\title{
ON THE DEFORMATION OF SURFACES OF TRANSLATION.
}

BY DR. BURKE SMITH.

(Read before the American Mathematical Society, December 29, 1904.)

IT is here proposed to find all surfaces of translation which may be deformed so that their generating lines remain generating lines throughout the deformation. The generating lines, $u=$ const., $v=$ const., of a surface of translation

(1) $x=f_{1}(u)+\phi_{1}(v), \quad y=f_{2}(u)+\phi_{2}(v), \quad z=f_{3}(u)+\phi_{3}(v)$

form a conjugate system, since $x, y, z$ satisfy an equation of the form *

$$
\frac{\partial^{2} \theta}{\partial u \partial v}=a \frac{\partial \theta}{\partial u}+b \frac{\partial \theta}{\partial v} \text {. }
$$

The square of the lineal element of (1) is,

$$
d s^{2}=E d u^{2}+2 F d u d v+G d v^{2},
$$

where

$$
E=\Sigma{f_{i}^{\prime}}^{2}, \quad F=\Sigma f_{i}^{\prime} \phi_{i}^{\prime}, \quad G=\Sigma{\phi_{i}^{\prime 2}}^{2} \quad(i=1,2,3)
$$

and the primes denote derivatives of $u$ and $v$ respectively.

Forming the symbols of Christoffel from (3) we have

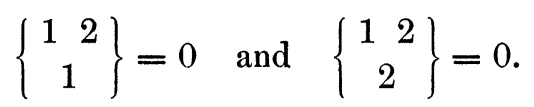

Conversely, if a surface $S$ is referred to a conjugate system of lines and if (4) is true, then $S$ must be a surface of translation, since its coördinates $x, y, z$ must satisfy

$$
\frac{\partial^{2} \theta}{\partial u \partial v}=\left\{\begin{array}{cc}
1 & 2 \\
1
\end{array}\right\} \frac{\partial \theta}{\partial u}+\left\{\begin{array}{cc}
1 & 2 \\
2
\end{array}\right\} \frac{\partial \theta}{\partial v}
$$

the integration of which gives (1) by virtue of (4). Hence we have the following

* Bianchi-Lukat : Vorlesungen über Differentialgeometrie, p. 110. 
Theorem. A surface of translation, when referred to its conjugate system of generating lines, is characterized by the equations (4).

Consider now the images on the Gauss sphere of a conjugate system of lines on any surface.

The relations between the symbols of Christoffel formed for a surface and for the corresponding Gauss sphere are as follows : *

$$
\left\{\begin{array}{cc}
1 & 2 \\
1
\end{array}\right\}=-\frac{D^{\prime \prime}}{D}\left\{\begin{array}{cc}
1 & 1 \\
2
\end{array}\right\}^{\prime} ; \quad\left\{\begin{array}{c}
1 \\
2
\end{array}\right\}=-\frac{D}{D^{\prime \prime}}\left\{\begin{array}{cc}
2 & 2 \\
1
\end{array}\right\}^{\prime}
$$

where the primes refer to the Gauss sphere and the second fundamental magnitudes of the surface are $D, 0, D^{\prime \prime}$.

Suppose now that $D \neq 0$ and $D^{\prime \prime} \neq 0$. Then from (5), if $\left\{\begin{array}{c}12 \\ 1\end{array}\right\}=0$ and $\left\{\begin{array}{c}12 \\ 2\end{array}\right\}=0$ we must also have,

$$
\left\{\begin{array}{cc}
1 & 1 \\
2
\end{array}\right\}^{\prime}=0 \text { and }\left\{\begin{array}{c}
2 \\
1
\end{array}\right\}^{\prime}=0
$$

and conversely. It follows $\dagger$ that the images on the Gauss sphere of the generating lines of a non-developable surface of translation are geodesics, and we may state the

THEOREM : The non-developable surfaces of translation are characterized by the fact that when they are referred to their generating lines, these lines have as images on the Gauss sphere the geodesics of the sphere.

When either $D=0$ or $\mathrm{D}^{\prime \prime}=0$, the surface is a developable surface. In the case of surfaces of translation, if $D^{\prime \prime}=0$ the equations of Codazzi require that $\partial D / \partial v=0$ and $\left\{\begin{array}{c}22 \\ 1\end{array}\right\}=0$. Hence, calculating $D$ and $D^{\prime \prime}$ from (1), we must have in this case

$$
\begin{array}{r}
f_{1}^{\prime}\left(\phi_{3}^{\prime} \phi_{2}^{\prime \prime}-\phi_{2}^{\prime} \phi_{3}^{\prime \prime}\right)+f_{2}^{\prime}\left(\phi_{1}^{\prime} \phi_{3}^{\prime \prime}-\phi_{3}^{\prime} \phi_{1}^{\prime \prime}\right)+f_{3}^{\prime}\left(\phi_{2}^{\prime} \phi_{1}^{\prime \prime}-\phi_{1}^{\prime} \phi_{2}^{\prime \prime}\right)=0, \\
\phi_{1}^{\prime}\left(f_{2}^{\prime \prime} f_{3}^{\prime}-f_{3}^{\prime \prime} f_{2}^{\prime}\right)+\phi_{2}^{\prime}\left(f_{1}^{\prime} f_{3}^{\prime \prime}-f_{3}^{\prime} f_{1}^{\prime \prime}\right)+\phi_{3}^{\prime}\left(f_{2}^{\prime} f_{1}^{\prime \prime}-f_{1}^{\prime} f_{2}^{\prime \prime}\right) \\
=U \sqrt{E G-F^{2}}, \quad\left\{\begin{array}{cc}
2 & 2 \\
1
\end{array}\right\}=0,
\end{array}
$$

where $U$ is a function of $u$ alone and the primes denote derivatives. These equations are satisfied when

* Bianchi-Iukat, 1. c., p. 135.

† Ibid., 1. c., p. 154. 
1905.] DEFORMATION OF SURFACES OF TRANSLATION. 189

$$
\phi_{3}^{\prime} \phi_{2}^{\prime \prime}-\phi_{2}^{\prime} \phi_{3}^{\prime \prime}=\phi_{1}^{\prime} \phi_{3}^{\prime \prime}-\phi_{3}^{\prime} \phi_{1}^{\prime \prime}=\phi_{2}^{\prime} \phi_{1}^{\prime \prime}-\phi_{1}^{\prime} \phi_{2}^{\prime \prime}=0,
$$

which leads to the cylindrical surfaces

$$
x=f_{1}(u)+c_{1} v, \quad y=f_{2}(u)+c_{2} v, \quad z=f_{3}(u)+c_{3} v,
$$

where $c_{i}=$ constant.

Bianchi has shown * that surfaces $S$ which may be deformed with preservation of a conjugate system are the associates of surfaces $S_{0}$ whose total curvature, in terms of the parameters of their asymptotic lines is of the form, $K=-[\phi(u)+\psi(v)]^{-2}$. We now proceed to determine the surfaces $S_{0}$ which are the associates of the surfaces of translation. Since a surface referred to a conjugate system of lines has the same spherical representation as its associate surface referred to its asymptotic lines, (5) may be interpreted not only for the surfaces of translation, but also for the surfaces to which they are associated. The following relations exist between the symbols of Christoffel formed for any surface referred to its asymptotic lines and for the corresponding Gauss sphere $: \dagger$

$$
\left\{\begin{array}{cc}
1 & 1 \\
2
\end{array}\right\}=-\left\{\begin{array}{cc}
1 & 1 \\
2
\end{array}\right\}^{\prime}, \quad\left\{\begin{array}{cc}
2 & 2 \\
1
\end{array}\right\}=-\left\{\begin{array}{cc}
2 & 2 \\
1
\end{array}\right\}^{\prime} .
$$

Then by (5), (6) and (7) the surfaces to which the surfaces of translation are associated are such that

$$
\left\{\begin{array}{cc}
1 & 1 \\
2
\end{array}\right\}=0 \text { and }\left\{\begin{array}{cc}
2 & 2 \\
1
\end{array}\right\}=0 .
$$

But (8) is the necessary and sufficient condition that the parametric lines should be geodesics, and hence the surface $S_{0}$ to which a surface of translation is associated is one on which both systems of parametric lines are at the same time asymptotic lines and geodesic lines. Since such lines are necessarily straight lines, the surface $S_{0}$ must be a doubly ruled surface, the two systems of parametric lines being the rectilinear generatrices of the surface. But since the only surface possessing two systems of straight lines lying wholly on the surface is a quadric, we may state the

Theorem. The surfaces to which the non-developable surfaces

* Bianchi-Lukat, p. 337.

+ Ibid., l. c., p. 127. 
of translation are associated are the quadric surfaces, and the rectilinear generatrices of the quadric surfaces correspond to the generating lines of the surfaces of translation. The non-developable surfaces of translation which may be deformed with preservation of generating lines are those surfaces, and only those, which are the associates of the quadric surfaces whose curvature, when expressed in terms of their asymptotic lines, is of the form

$$
K=-[\phi(u)+\psi(v)]^{-2}
$$

The quadrics whose curvature is of the above form will now be determined. Consider first a central quadric. The equations of a central quadric in terms of the parameters of the asymptotic lines are

$$
x=a\left(\frac{1+u v}{u+v}\right), \quad i y=b\left(\frac{1-u v}{u+v}\right), \quad z=c\left(\frac{u-v}{u+v}\right) .
$$

By direct calculation from (10) we have for the total curvature

$$
K=\frac{a^{2} b^{2} c^{2}(u+v)^{4}}{\left[a^{2} b^{2}(u-v)^{2}-a^{2} c^{2}(1-u v)^{2}+b^{2} c^{2}(1+u v)^{2}\right]^{2}} .
$$

The only case in which $K$ is of the required form is when $b^{2}=c^{2}=a^{2}$, and the quadric then reduces to a sphere, for which $K=1 / a^{2}$.

Consider next the paraboloids. The equations of the paraboloids in terms of the parameters of the asymptotic lines are

$$
x=\frac{u+v}{2 \sqrt{l}}, \quad y=\frac{u-v}{2 i \sqrt{m}}, \quad 2 z=u v
$$

where $l$ and $m$ are constants.

From (11) we have

$$
K=\frac{16 l m}{\left[4+(l-m) u^{2}+2(l+m) u v+(l-m) v^{2}\right]^{2}},
$$

and hence $K$ is here of the required form when and only when $l=-m$, in which case (11) are the equations of the equilateral hyperbolic paraboloid.

Thus the only quadric surfaces whose curvature expressed in terms of the parameters of the asymptotic lines is of the form (9) are the sphere and the equilateral hyperbolic para- 
boloid. Now the associate surfaces of a sphere are the minimum surfaces, ${ }^{*}$ and the associate surfaces of the equilateral hyperbolic paraboloid are the surfaces of translation whose generating lines are plane and lie in perpendicular planes. $\dagger$ Hence we may state the

THEOREM : The only non-developable surfaces of translation which may be deformed so that their generating lines remain generating lines throughout the deformation are the minimum surfaces and those surfaces of translation whose two systems of generating lines are plane and lie in perpendicular planes.

Purdue University, October, 1904.

\section{THE INTERNATIONAL CONGRESS OF MATHE- MATICIANS AT HEIDELBERG.}

The International Congress of 1904 was held at Heidelberg, August 8 to 13, under the efficient management of the Deutsche Mathematiker-Vereinigung. The attendance, 336, showed continued advance in comparison with the preceding congresses of Zürich and Paris, the proceedings and papers were of much interest and in some cases of notable importance, and the local arrangements, both for meetings and for hospitable welcome, were generous and admirable. One could have wished only for an increase of the day's normal twenty-four hours, or a distribution of sectional meetings which would render it less difficult to attend more than one section. The programme for the week was in its main outlines as follows :

Monday, August 8.- Informal gathering at the City Hall, with an address of welcome by Professor Moritz Cantor.

Tuesday, August 9.-General session. Addresses of welcome by the son of the Grand Duke of Baden and other official representatives. Address in commemoration of Jacobi by Professor Königsberger. Organization of the six sections. Banquet in the City Hall.

Wednesday, August 10.-Meetings of the sections. Reception at the grand ducal palace at Schwetzingen.

* See Eisenhart, Bulletin, March, 1902, p. 243.

† Bianchi-Lukat, p. 338 . 